\title{
An Integrated Procedure for Shape Optimization Applied to Fluid Flow
}

L.M. STEFFENS ${ }^{1}$, M.K. ALVES ${ }^{2}$, Centro Tecnológico, Departamento de Engenharia Mecânica, POSMEC, UFSC, 88040-900 Florianópolis, SC, Brasil.

\begin{abstract}
This work proposes an integrated numerical procedure for the shape optimization of fluid flow problems. The procedure is named integrated since it combines various distinct modules for the solution to the problem, such as: geometric modeling, mesh generation for finite elements, nonlinear analysis of the fluid flow, sensitivity analysis, mathematical programming and shape optimization. The problem consists in the optimization of the contour shapes, aiming the reduction of the viscous dissipation produced by the flow around a given body or channel.
\end{abstract}

\section{Introduction}

Many are the applications of shape optimization to fluid flow problems. These applications are especially important in the aerospace and automotive engineering field and also in the design of valves and hydraulic pumps. In general, engineers are interested in reducing the drag force on the wing of a plane or on a vehicle, or in reducing the viscous dissipation in hydraulic valves, pipes, etc...

The two-dimensional fluid flow problem is assumed to be incompressible and steady state and is modeled by the Navier-Stokes equations. The approximate solution to the flow problem is obtained by applying the Galerkin Finite Element method. In order to satisfy the Brezzi-Babuska condition a T7/C3 interpolation scheme is employed (triangular element with seven nodes for the velocity and three for the pressure). The design variables of the optimization problem are the position of the key-points, which describe the contour of the domain that is parameterized by segments of cubic B-splines. The discrete optimization problem considers the minimization of the viscous dissipation of the flow and is subjected to a volume and side constraints, and is formulated through the application of the Augmented Lagrangian problem, reducing the problem to the solution of a sequence of box constrained optimization problems. An efficient solution to this class of optimal shape problems depends strongly on the strategy utilized in the sensitivity analysis of the fluid flow equation, which is necessary for the determination of the gradient of the objective function and constraints. Here, this is achieved with the use of an adjoint method for the determination of the sensitivity analysis and computation of the required gradients. In order to investigate the performance of the proposed procedure, one solves some simple nozzle optimization problems.

\footnotetext{
${ }^{1}$ Lindaura Maria Steffens, Ms. Eng. (2005, UFSC - Brasil) - lindaura@emc.ufsc.br

${ }^{2}$ Marcelo Krajnc Alves, Ph.D. (1991, Univ. Michigan - EUA) - krajnc@emc.ufsc.br.
} 


\section{Equation of Navier-Stokes}

Strong Formulation: The flow problem consists in solving the Navier-Stokes equations [9], and can be stated as: Let $\Omega \subset \mathfrak{R}^{2}$ be a bounded fluid domain with boundary $\Gamma$. The problem consists in finding $\vec{u}(\vec{x})$ and $p(\vec{x}), \forall \vec{x} \in \Omega \cup \Gamma$, so that:

$$
\begin{gathered}
(\nabla \vec{u}(\vec{x})) \vec{u}(\vec{x})-2 \boldsymbol{\nu} \operatorname{div}(D(\vec{u}(\vec{x})))+\frac{1}{\rho} \nabla p(\vec{x})=\vec{b}(\vec{x}) \text { in } \Omega \\
\operatorname{div}(\vec{u}(\vec{x}))=0 \quad \text { in } \Omega
\end{gathered}
$$

in which $\vec{x}$ represents the position of the design variables, $\vec{u}$ the velocity vector, $p$ the fluid pressure, $\boldsymbol{\nu}$ the dynamical viscosity, $\rho$ the specific density, $D$ the symmetric part of the velocity gradient and $\vec{b}$ the body force vector.

The Equations in (2.1) are subjected to Dirichlet and Neumann boundary conditions, which are given by:

$$
\begin{gathered}
\vec{u}(\vec{x})=\tilde{\mathbf{u}}(\vec{x}) \quad \text { at } \quad \Gamma_{\mathbf{u}} \\
2 \boldsymbol{\nu} D(\vec{u}(\vec{x})) \cdot \widehat{n}-\frac{1}{\rho} p(\vec{x}) \widehat{n}=\vec{h}(\vec{x}) \quad \text { at } \quad \Gamma_{\mathbf{t}},
\end{gathered}
$$

where $\Gamma_{\mathbf{u}}$ and $\Gamma_{\mathbf{t}}$ are the parts of the boundary subjected to a prescribed velocity and surface traction conditions respectively.

Weak Formulation: Let $\operatorname{Kin}_{u}=\left\{\vec{u}(\vec{x}) \in\left[H^{1}(\Omega)\right]^{2} \mid \vec{u}(\vec{x})=\tilde{\mathbf{u}}(\vec{x})\right.$ at $\left.\Gamma_{\mathbf{u}}\right\}, \operatorname{Var}_{u}=$ $\left\{\vec{v}(\vec{x}) \in\left[H^{1}(\Omega)\right]^{2} \mid \vec{v}(\vec{x})=\overrightarrow{0}\right.$ at $\left.\Gamma_{\mathbf{u}}\right\}, \operatorname{Kin}_{p}=\left\{p(\vec{x}) \in L^{2}(\Omega)\right\}$ and $\operatorname{Var}_{p}=\{\hat{p}(x) \in$ $\left.L^{2}(\Omega)\right\}$, where the linear spaces $H^{1}$ and $L^{2}$ are defined in [7]. The weak formulation of the problem can be stated as: Determine $(\vec{u}, p) \in \mathbf{K i n}_{u} \times \mathbf{K i n}_{p}$, such that:

$$
\begin{gathered}
\int_{\Omega}\left\{(\nabla \vec{u}) \vec{u}-2 \boldsymbol{\nu} \operatorname{div}(D(\vec{u}))+\frac{1}{\rho} \nabla p-\vec{b}\right\} \cdot \vec{v} d \Omega-\int_{\Omega}\left\{\frac{1}{\rho} \operatorname{div}(\vec{u})\right\} \hat{p} d \Omega=0 \\
\forall(\vec{v}, \hat{p}) \in \operatorname{Var}_{u} \times \operatorname{Var}_{p} .
\end{gathered}
$$

Now, adding a shock capturing term, applying the divergence theorem [1], considering the boundary conditions defined in Eq.(2.2), and doing some algebra, one is able to reformulate the weak form as follows: Determine $(\vec{u}, p) \in \mathbf{K i n}_{u} \times \mathbf{K i n}_{p}$, such that:

$$
\begin{aligned}
& \langle\nabla(\vec{u}), \vec{v}\rangle_{\Omega}+2 \boldsymbol{\nu}\langle D(\vec{u}), D(\vec{v})\rangle_{\Omega}-\frac{1}{\rho}\langle p, \operatorname{div}(\vec{v})\rangle_{\Omega}-\frac{1}{\rho}\langle\operatorname{div}(\vec{u}), \hat{p}\rangle_{\Omega}+ \\
& \langle\operatorname{div}(\vec{u}), \boldsymbol{\delta} \operatorname{div}(\vec{v})\rangle_{\Omega}=\langle\vec{b}, \vec{v}\rangle_{\Omega}+\langle\vec{h}, \vec{v}\rangle_{\Gamma_{\mathbf{t}}}, \quad \forall(\vec{v}, \hat{p}) \in \operatorname{Var}_{u} \times \operatorname{Var}_{p} .
\end{aligned}
$$

where $\langle f(\vec{x}), g(\vec{x})\rangle=\int_{\Omega}\{f(\vec{x}) \cdot g(\vec{x})\} d \Omega$, denotes the inner product of the arbitrary functions. The parameter $\boldsymbol{\delta}$ in the shock capturing term is defined in [3].

Discretization of the Problem: At this point, one performs a partition of the domain $\Omega$ into elements $\Omega_{e}$, in which the velocity and pressure fields are interpolated as:

$$
u=\left[\mathbb{N}_{i}\right] \vec{q}_{e}^{u} ; \quad v=\left[\mathbb{N}_{i}\right] \vec{q}_{e}^{v} ; \quad p=\left[\mathbb{N}_{i}\right] \vec{q}_{e}^{p} .
$$

Here, $\left[\mathbb{N}_{i}\right]_{i=\vec{u}, p}$ is the vector containing the classical finite element interpolation functions, $\vec{q}_{e}{ }^{u}, \vec{q}_{e}^{v}$ and $\vec{q}_{e}^{p}$ represent the vector of nodal velocities and pressures. 
The discretization of Eq. (2.3) is done as:

$$
\begin{aligned}
& \langle(\nabla \vec{u}) \vec{u}, \vec{v}\rangle_{\Omega_{\mathbf{e}}}=\left[\int_{\Omega_{\mathbf{e}}}\left[\mathbb{N}^{d i s p}\right]^{T}\left[\mathbb{N}^{g r a d u}(\vec{u})\right] d \Omega\right] \overrightarrow{q_{e}} \cdot \hat{q}_{e}=\left[\mathbb{k}_{e}^{\nabla u u}(\vec{u})\right] \vec{q}_{e} \cdot \hat{q}_{e}, \\
& 2 \boldsymbol{\nu}\langle D(\vec{u}), D(\vec{v})\rangle_{\Omega_{\mathbf{e}}}=\left[\int_{\Omega_{\mathbf{e}}}\left[\mathbb{B}^{\mathbf{v}}\right]^{T}\left[\mathbb{H}^{\mathbf{v}}\right]\left[\mathbb{B}^{\mathbf{v}}\right] d \Omega\right] \vec{q}_{e} \cdot \hat{q}_{e}=\left[\mathbb{k}_{e}^{\mathbf{v}}\right] \vec{q}_{e} \cdot \hat{q}_{e} \\
& \frac{1}{\rho}\langle p, \operatorname{div}(\vec{v})\rangle_{\Omega_{\mathbf{e}}}=\frac{1}{\rho}\left[\int_{\Omega_{\mathbf{e}}}\left[\left[\mathbb{B}^{d i v}\right] \otimes\left[\mathbb{N}^{\text {press }}\right]\right] d \Omega\right] \vec{q}_{e} \cdot \hat{q}_{e}=\left[\mathbb{k}_{e}^{\text {press }}\right] \vec{q}_{e} \cdot \hat{q}_{e} \\
& \frac{1}{\rho}\langle\operatorname{div}(\vec{u}), \hat{p}\rangle_{\Omega_{\mathbf{e}}}=\frac{1}{\rho}\left[\int_{\Omega_{\mathbf{e}}}\left[\left[\mathbb{N}^{\text {press }}\right] \otimes\left[\mathbb{B}^{d i v}\right]\right] d \Omega\right] \vec{q}_{e} \cdot \hat{q}_{e}=\left[\mathbb{k}_{e}^{\hat{p}}\right] \vec{q}_{e} \cdot \hat{q}_{e}, \\
& \left.\langle\operatorname{div}(\vec{u}), \boldsymbol{\delta} \operatorname{div}(\vec{v})\rangle_{\Omega_{\mathbf{e}}}=\left[\int_{\Omega_{\mathbf{e}}} \boldsymbol{\delta}\left[\left[\mathbb{B}^{d i v}\right] \otimes\left[\mathbb{B}^{\operatorname{div}}\right]\right] d \Omega\right] \vec{q}_{e} \cdot \hat{q}_{e}=\mathbb{k}_{e}\right] \vec{q}_{e} \cdot \hat{q}_{e} \\
& \langle\vec{b}, \vec{v}\rangle_{\Omega_{\mathbf{e}}}=\left[\int_{\Omega_{\mathbf{e}}}\left[\mathbb{N}^{d i s p}\right]^{T} \vec{b} d \Omega\right] \cdot \hat{q}_{e}=\left[F_{e}^{b}\right] \cdot \hat{q}_{e}, \\
& \langle\vec{h}, \vec{v}\rangle_{\Gamma_{\mathbf{t}} \cap \Omega_{\mathbf{e}}}=\left[\int_{\Gamma_{\mathbf{t}} \cap \Omega_{\mathbf{e}}}\left[\mathbb{N}^{d i s p}\right]^{T} \vec{h} d \Gamma\right] \cdot \hat{q}_{e}=\left[F_{e}^{t}\right] \cdot \hat{q}_{e},
\end{aligned}
$$

where:

$$
\begin{aligned}
{\left[\mathbb{N}^{\text {gradu }}(\vec{u})\right] } & =\left[\begin{array}{ccc}
u \mathbb{N}_{u, x}+v \mathbb{N}_{u, y} & 0 & 0 \\
0 & u \mathbb{N}_{u, x}+v \mathbb{N}_{u, y} & 0
\end{array}\right], \\
{\left[\mathbb{N}^{\text {disp }}\right] } & =\left[\begin{array}{ccc}
\mathbb{N}_{u} & 0 & 0 \\
0 & \mathbb{N}_{u} & 0
\end{array}\right], \\
{\left[\mathbb{H}^{\mathbf{v}}\right] } & =\left[\begin{array}{ccc}
2 \boldsymbol{\nu} & 0 & 0 \\
0 & 2 \boldsymbol{\nu} & 0 \\
0 & 0 & \boldsymbol{\nu}
\end{array}\right],\left[\mathbb{B}^{\mathbf{v}}\right]\left[\begin{array}{ccc}
\mathbb{N}_{u, x} & 0 & 0 \\
0 & \mathbb{N}_{u, y} & 0 \\
\mathbb{N}_{u, y} & \mathbb{N}_{u, x} & 0
\end{array}\right], \\
{\left[\mathbb{N}^{\text {press }}\right] } & =\left\{\begin{array}{ccc}
0 & 0 & \mathbb{N}_{p}
\end{array}\right\},\left[\mathbb{B}^{\text {div }}\right]=\left\{\begin{array}{lll}
\mathbb{N}_{u, x} & \mathbb{N}_{u, y} & 0
\end{array}\right\} .
\end{aligned}
$$

Once determined the contribution of each element in the discretized weak formulation, one assembles the element vectors and matrices into the global vectors and matrices and form the global system of nonlinear equations, given by:

$$
\left\{\begin{array}{c}
\vec{q} \Longleftarrow \bigcup_{e=1}^{n e} \vec{q}_{e} ; \\
{[\mathbb{K}(\vec{q})] \Longleftarrow \underset{e=1}{\mathbf{A}_{e}}\left\{\left[\mathbb{k}_{e}^{g r d u}(\vec{u})\right]+\left[\mathbb{k}_{e}^{\mathbf{v}}\right]-\left[\mathbb{k}_{e}^{p r e s s}\right]-\left[\mathbb{k}_{e}^{p r e s s}\right]^{T}+\left[\mathbb{k}_{e}^{\boldsymbol{\delta}}\right]\right\}} \\
{\left[\vec{F}^{e x t}\right] \Longleftarrow \underset{e=1}{\mathbf{A}}\left\{\left[F_{e}^{b}\right]+\left[F_{e}^{t}\right]\right\} ;}
\end{array}\right.
$$

in which $n e$ is the number of elements and $\underset{e=1}{n e}\{\cdot\}$ is an assemblage operator, responsible for the assemblage of the global equation system. Notice that, since $\left[\mathbb{k}_{e}^{\text {gradu }}(\vec{u})\right]$ is a function of $\vec{u}$, the resulting global discrete problem is nonlinear and may be expressed as:

$$
[\mathbb{K}(\vec{q})] \vec{q}=\left[\vec{F}^{e x t}\right] .
$$

In order to solve this set of nonlinear equations, one applies Newton's method. 
Flow analysis: In order to validate the proposed procedure, the classic square lid-driven cavity problem was selected, see ([4],[8]). The problem consists on an incompressible flow confined into a square cavity, whose upper face is moved with a constant prescribed horizontal velocity. (See Fig. 1).

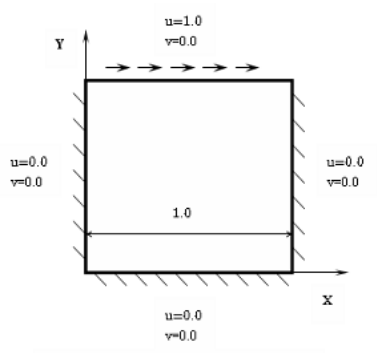

Figure 1: Square lid-driven cavity.

Figure 2 shows a comparative graph of the velocity profiles calculated in this work (uLM and vLM) with the data presented in [4] (uEC and vEC) for the case of $\operatorname{Re}=2500$.
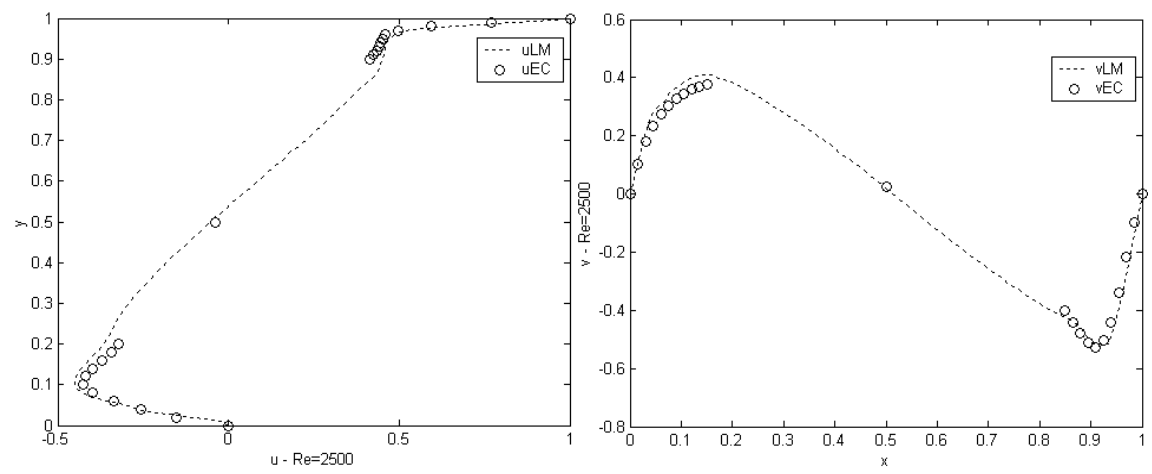

Figure 2: Profile of velocity components $\mathrm{u}$ and $\mathrm{v}$ along the central vertical and

\section{Optimization}

Contour definition: Different techniques may be employed for the parameterization of the boundary of the domain to be optimized, such as the use of macroelements, or polynomial functions. However, these techniques present some numerical problems when used with complex geometries. On the other side, a considerable advance was achieved with the use of parametric splines representations of boundary contours. They allow a local control of the curve and require few input data. Among the different types of parametric splines, there is the cubic B-splines, which is the most use since they attend the majority of practical requirements, see [5] and [6]. Due to the simplicity and the versatility, this work adopted the parametric 
cubic B-splines that may be represented as:

$$
\vec{p}(t)^{T}=\left\{p_{x}(t), p_{y}(t), p_{z}(t)\right\}=\vec{T}\left[\mathbb{M}_{\mathbf{B S}}\right] \vec{B}_{\mathbf{B S}},
$$

in which

$\vec{T}=\left\{t^{3} t^{2} t 1\right\},\left[\mathbb{M}_{\mathbf{B S}}\right]=\frac{1}{6}\left[\begin{array}{rrrr}-1 & 3 & -3 & 1 \\ 3 & -6 & 3 & 0 \\ -3 & 0 & 3 & 0 \\ 1 & 4 & 1 & 0\end{array}\right], \vec{B}_{\mathbf{B S}}=\left\{\begin{array}{c}\vec{b}_{i-1} \\ \vec{b}_{i} \\ \vec{b}_{i+1} \\ \vec{b}_{i+2}\end{array}\right\} i \in[1: K-1]$.

Here, $\vec{b}_{i-1}, \vec{b}_{i}, \vec{b}_{i+1}$ and $\vec{b}_{i+2}$ are the position vectors of the vertices of control $\mathrm{B}_{i-1}, \mathrm{~B}_{i}, \mathrm{~B}_{i+1}$ and $\mathrm{B}_{i+2}$, of the representative polygons that determine each curve segment and $(K-1)$ is the number of segment curves. (See Fig. 3).

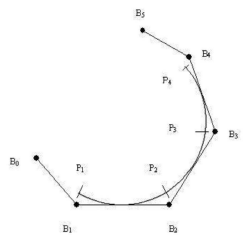

Figure 3: Cubic B-spline segment passing through a set of key points.

The design variables of the optimization problem are the position vectors of the key points that define the curve segments, denoted by $\vec{s}$, which describe the domain contour by the cubic B-splines segments.

Objective Function: is given by the dissipation of the viscous flow integrated in the entire domain $\Omega$ :

$f(\vec{u}(\vec{x}(\vec{s})), \vec{s})=2 \boldsymbol{\nu}\langle D(\vec{u}(\vec{x}(\vec{s}))), D(\vec{u}(\vec{x}(\vec{s})))\rangle_{\Omega(\vec{s})}=2 \boldsymbol{\nu} \int_{\Omega(\vec{s})} D(\vec{u}(\vec{x}(\vec{s}))) \cdot D(\vec{u}(\vec{x}(\vec{s}))) d \Omega$

The finite element discretization of Eq.(3.1) is given by:

$f(\vec{q}(\vec{s}), \vec{s})=\left[\mathbb{K}^{\mathbf{v}}(\vec{s})\right] \vec{q}(\vec{s}) \cdot \vec{q}(\vec{s}), \quad\left[\mathbb{K}^{\mathbf{v}}(\vec{s})\right]=\sum_{e=1}^{n e}\left[\mathbb{k}_{e}^{\mathbf{v}}(\vec{s})\right]=\sum_{e=1}^{n e}\left[\int_{\Omega_{\mathbf{e}}}\left[\mathbb{B}^{\mathbf{v}}\right]^{T}\left[\mathbb{H}^{\mathbf{v}}\right]\left[\mathbb{B}^{\mathbf{v}}\right] d \Omega\right]$.

Definition of the optimization problem: The discrete optimization problem may be stated as:

Find $\vec{s} \in S$ solution of

$$
\min _{s / a} f(\vec{q}(\vec{s}), \vec{s}) \quad s / a \vec{g}(\vec{s}) \leq 0, \forall \vec{s} \in S .
$$

in which $S=\left\{\vec{s} \in \mathfrak{R}^{n} \mid s_{i}^{\text {inf }} \leq s_{i} \leq s_{i}^{\text {sup }}, i=1, . ., n\right\}$ represents the set of lateral constraints, $\vec{g}(\vec{s})=\Omega(\vec{s})-\Omega^{\text {sup }}$ a volumetric constraint and $\vec{q}(\vec{s})$ the vector of nodal velocities and pressure associated with the finite element discretization of the flow problem. 
By Applying the Augmented Lagrangian method, one is able to compute an approximated solution to Eq.(3.3) by solving a sequence of bound constrained problems, defined as:

Determine $\vec{s}^{*} \in \mathfrak{R}^{n}$ so that $\vec{s}^{*}=\lim _{k \rightarrow \infty} \vec{s}_{k}^{*}$, where $\vec{s}_{k}^{*}$ is solution of the problem:

Given $\vec{\mu}^{k} \in \mathfrak{R}^{m}$ and $\varepsilon^{k}>0$ determine $\vec{s}_{k}^{*}$, which solves:

$$
\min \chi\left(\vec{s}, \vec{\mu}^{k}, \varepsilon^{k}\right) \quad s / a \vec{s} \in S .
$$

where

$$
\begin{aligned}
\chi\left(\vec{s}, \vec{\mu}^{k}, \varepsilon^{k}\right) & =f(\vec{s})+\frac{1}{2 \varepsilon^{k}} \sum_{j=1}^{m} \Psi_{j}\left(g_{j}(\vec{s}), \varepsilon^{k} \vec{\mu}_{j}^{k}\right) ; \\
\Psi_{j}\left(g_{j}(\vec{s}), \varepsilon^{k} \vec{\mu}_{j}^{k}\right) & =\left\{\begin{array}{l}
{\left[2 \varepsilon^{k} \vec{\mu}_{j}^{k}+g_{j}(\vec{s})\right] g_{j}(\vec{s}) \text { if } g_{j}(\vec{s}) \geq-\varepsilon^{k} \vec{\mu}_{j}^{k} ;} \\
-\left(\varepsilon^{k} \vec{\mu}_{j}^{k}\right)^{2} \text { if } g_{j}(\vec{s})<-\varepsilon^{k} \vec{\mu}_{j}^{k} .
\end{array}\right.
\end{aligned}
$$

The Lagrange multiplier vector $\vec{\mu}$ and the penalty parameter $\varepsilon>0$, are then updated in order to completely define the iterative process. The actualization of these parameters is given in [8]. The convergence criterion used in the work is the Kuhn-Tucker optimality criterion, [2].

Sensitivity Analysis:The sensitivity analysis requires the determination of the gradient of some response function, with respect to the design variables. Generally, these functions are implicit and non linear with respect to the design variables, which makes their gradient difficult to determine.

The objective function, defined in Eq. (3.2), depends explicitly and implicitly on $\vec{s}$. The partial derivative of $f$ with respect to $s_{j}$ is given by:

$$
\frac{d f}{d s_{j}}=\frac{\partial f}{\partial s_{j}}+\frac{\partial f}{\partial \vec{q}} \frac{d \vec{q}}{d s_{j}}, j=1, . ., j=\text { number of design variables. }
$$

The derivatives of $f$ with respect to $\vec{q}$ and $s_{j}$ are simple to be determined. In general, the determination of $d \vec{q} / d s_{j}$ is more complex. One way to circumvent the necessity of determining $d \vec{q} / d s_{j}$ is to apply the adjoint method. In this case, the objective function is redefined by the introduction of the adjoint vector $\vec{\lambda}$, and denoted by $\hat{f}$, i.e.,

$$
\hat{f}=f+\langle\vec{\lambda}, \vec{R}\rangle
$$

where $\vec{R}$ is the residual vector defined in the discrete form of the solution of the non linear flow problem. Thus, since in the equilibrium condition $\vec{R}=0$, one may choose $\vec{\lambda}$ arbitrarily since $\langle\vec{\lambda}, \vec{R}\rangle=0$. The gradient of the modified objective function (3.4) is then given by:

$$
\frac{d \hat{f}}{d s_{j}}=\frac{d f}{d s_{j}}+\left\langle\vec{\lambda}, \frac{d \vec{R}}{d s_{j}}\right\rangle=\frac{\partial f}{\partial s_{j}}+\left\langle\vec{\lambda}, \frac{\partial \vec{R}}{\partial s_{j}}\right\rangle+\left\langle\frac{\partial f}{\partial \vec{q}}+\vec{\lambda}\left[\mathbf{K}_{\mathbf{T}}\right], \frac{d \vec{q}}{d s_{j}}\right\rangle .
$$

in which $\left[\mathbf{K}_{\mathbf{T}}\right]=\partial \vec{R} / \partial \vec{q}$, where $\left[\mathbf{K}_{\mathbf{T}}\right]$ is the tangent matrix obtained in the solution of the fluid flow problem, when solved by Newton's method. Now, since $\vec{\lambda}$ can be 
chosen arbitrarily, it can be selected conveniently in order to eliminate the necessity of computing $d \vec{q} / d s_{j}$. Hence, $\vec{\lambda}$ is selected so that it is the solution of

$$
\left[\mathbf{K}_{\mathbf{T}}\right]^{T} \vec{\lambda}=-\frac{\partial f}{\partial \vec{q}} .
$$

Introducing the solution $\vec{\lambda}^{*}$ of Eq.(3.6) into Eq. (3.5), one derives:

$$
\frac{d \hat{f}}{d s_{j}}=\frac{\partial f}{\partial s_{j}}+\left\langle\vec{\lambda}^{*}, \frac{\partial \vec{R}}{\partial s_{j}}\right\rangle .
$$

Thus, in order to compute the gradient of the modified objective function, one computes the adjoint vector, by solving Eq.(3.6). Here, one notices that the tangent matrix $\left[\mathbf{K}_{\mathbf{T}}\right]$ is known since it was already computed in the solution of the flow problem.

\section{Problem Cases}

Consider the problems illustrated in Fig. 4, representing the flow of an incompressible fluid in a divergent channel, subjected to a prescribed velocity profile in the inlet, a traction free surface in the outlet, a non slip condition on the wall and a symmetry condition on the upper boundary. The cases to be considered are:

a) The optimization of a divergent channel;

b) The optimization of a divergent channel with an obstacle.

The problem consists in finding the optimal shape of the domain in order to minimize the total dissipation of the flow, subjected to lateral constraints and a volume constraint. Figures $4 \mathrm{a}$ and $4 \mathrm{~b}$ show the geometries to be optimized, while Figures $5 \mathrm{a}$ and $5 \mathrm{~b}$ illustrate the definition of the segments of the parameterized B-splines used to describe the domain boundary.

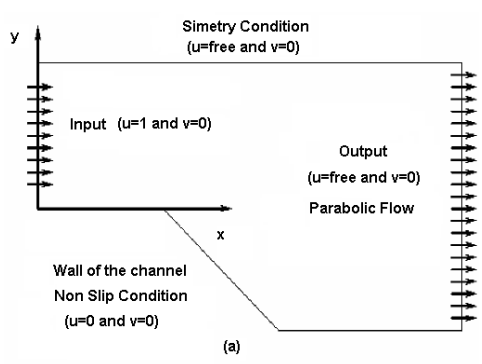

Figure 4: a) Ramp to be optimized.

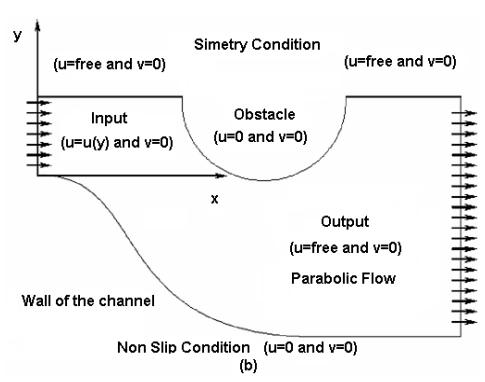

b) Diffuser and obstacle to be optimized.

In case a) the contour is described by 9 key points, 6 cubic B-splines segments, having 8 design variables defining the optimization problem and the corresponding degrees of freedom of each such key point, i.e., the form of their movement. In case b) the boundary is described by 12 key points, 6 cubic B-splines segments, having 9 design variables. Notice that, the design variables are concentrated in the segments 


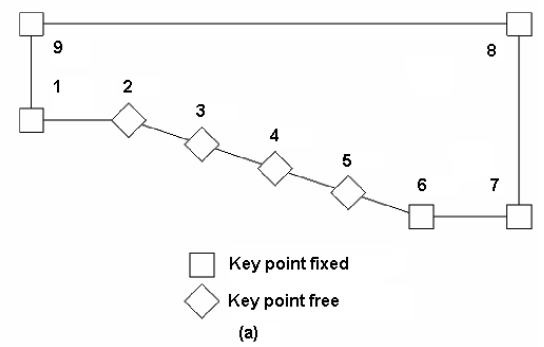

Figure 5: a) Definition of the key points.

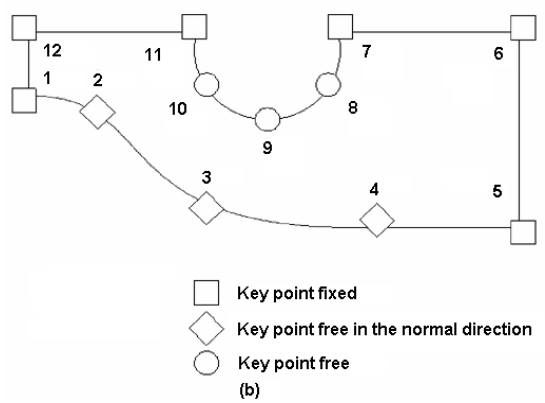

b) Definition of the key points.

that represent the regions to be optimized, where lateral restrictions are imposed. The volume constraint imposed in both cases allows an increase of the initial volume to at most $10 \%$. The optimization analysis was performed for a flow with Reynolds of 100 , based on the inlet size and prescribed velocity and a tolerance of $10^{-4}$ for the Newton's iterative method.

Some of the results obtained in the flow analysis, for the optimal shape configuration, are illustrated in Fig. 6, for the fluid velocity field, and Fig. 7, for the fluid pressure results.
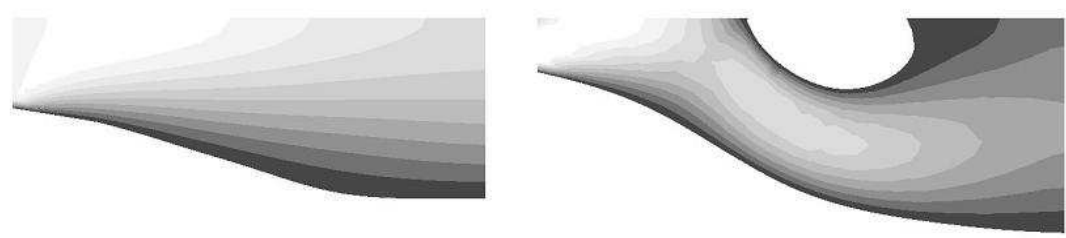

Figure 6: Euclidean norm of the velocity.
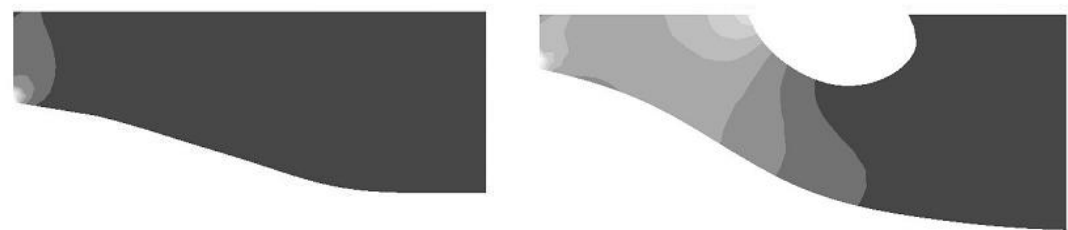

Figure 7: Pressure field.

The graphs in Fig. 8 show the initial and optimal position of the boundary contour for both diffuser cases, enabling a proper comparison between the initial and the final optimal shape.

The results obtained for the reduction of the flow dissipations were significant. In case a) the reduction was of approximately $28 \%\left(f_{\text {initial }}=1.20751\right.$ and $\left.f_{\text {otimal }}=0.79565\right)$, while in case b) was of approximately $55 \%\left(f_{\text {initial }}=2.66943\right.$ and $\left.f_{\text {otimal }}=1.21596\right)$. 

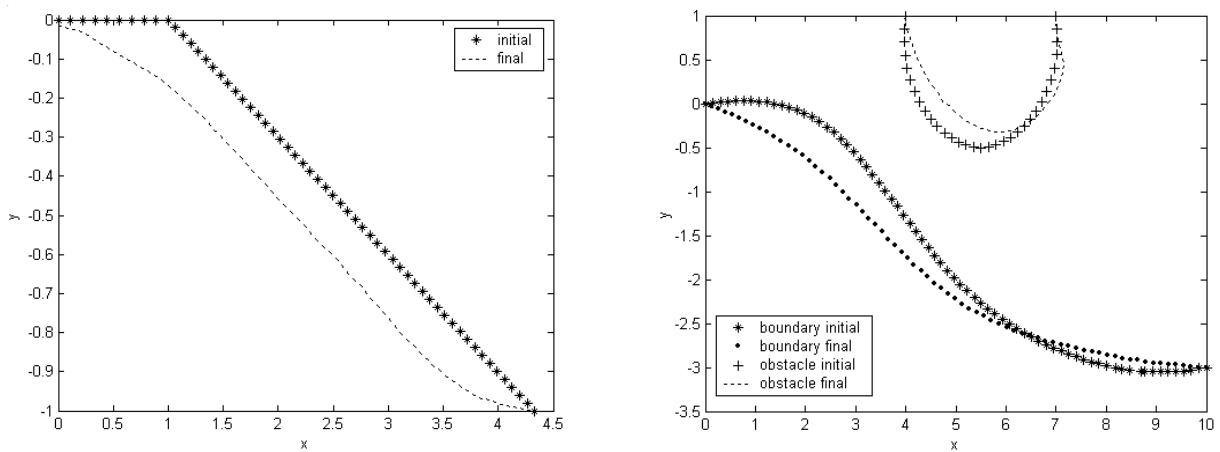

Figure 8: Position of the rampa, boundary at the wall and obstacle

Analysing the initial and final optimal shapes of the diffuser, one can notice a brodening of the channel in the ramp region causing a decrease of the flow velocity and of the velocity gradient, reducing in this way the value of the objective function. In the case of a diffuser with an obstacle, the optimal shape presents an obstacle with a shape that seemed to be dragged by the flow, while satisfying the constraints, causing in this way a reduction of the influence of the obstacle to the flow, implying in a reduction of the objective function. These results support the normal intuition that we would expect to have in these flow problems.

\section{Conclusions}

The discretization adopted by using the T7/C3 triangular element, for the solution of the flow problem, have shown to be adequate since they satisfy the BrezziBabuska condition with no need of additional terms. This simplifies considerably the work necessary to compute the sensitivity of the response of the flow problem with respect to the design variables.

The parametric representation of the boundary by cubic B-splines have shown to be very effective, since they not only allowed a significant reduction of the objective function but also, due to their simplicity of use and their ability to control locally the boundary, required a small number of design variables to obtain these results. By using cubic B-splines, it's possible to represent smoothly any contour, with irregular boundaries and with complex obstacles, in a natural and simple way, requiring a few input data parameters, what simplifies the handling and control of the key points that are used to describe the countour.

Resumo. Este trabalho propõe um procedimento numérico integrado para problemas que envolvem a otimização de forma aplicada ao escoamento de fluidos. O procedimento é denominado integrado porque reúne diversos módulos distintos para o tratamento do problema, como modelagem geométrica, geração de malhas por elementos finitos, análise não-linear do escoamento, análise de sensibilidade, programação matemática e otimização de forma. O problema a ser solucionado neste trabalho consiste na otimização de forma de contornos, visando à redução da dissipação viscosa decorrente do escoamento em torno de um dado corpo ou em um canal. 


\section{References}

[1] R. Aris, "Vectors, Tensors, and the Basic Equations of Fluid Mechanics", Dover, New York, 1989.

[2] J.S. Arora, "Introduction to Optimum Design", McGraw-Hill, New York, 1989.

[3] R. Codina, On Stabilized Finite Element Methods for Linear Sistems of Convection-Diffusion-Reaction Equations, Comp. Methods Appl. Mech. Eng., 188 (2000), 61-82.

[4] E. Erturk, T.C. Corke, Numerical Solutions of 2-D Steady Incompressible Driven Cavity Flow at High Reynolds Numbers, International Journal for Numerical Methods in Fluids, 48, (2005), 747-774.

[5] E. Hinton, J. Sienz, Reliable Structural Optimization with Error Estimation, Adaptivity and Robust Sensitivity Analysis, Computers \& Structures, 4 (1997), $31-63$.

[6] M.E. Mortenson, "Geometric Modeling", John Wiley \& Sons, New York, 1997.

[7] B.D. Reddy, "Introductory Functional Analysis with Applications to Boundary Value Problems Finite Elements", Springer, New York, 1997.

[8] L.M. Steffens, "Desenvolvimento de uma Metodologia Integrada para Otimização de Forma de Mecânica de Fluidos", Dissertação de Mestrado, EMC, UFSC, Florianópolis, SC, 2005.

[9] R. Temam, "Navier-Stokes Equations", Elsevier Science Publishers Company, Inc., New York, 1991. 